\title{
Papers
}

\section{The borderline cervical smear: colposcopic and biopsy outcome}

\author{
Awatif Al-Nafussi, Gemma Rebello, Raja Al-Yusif, Euphemia McGoogan
}

\begin{abstract}
Aims-To review the outcome of women referred with smears showing borderline nuclear change (BNC), and to determine any differences in outcome if BNC was persistent, preceded by dyskaryosis, or followed treatment for cervical intraepithelial neoplasia (CIN). In addition, to determine criteria that might permit delineation of a BNC subtype, predictive of CIN.

Methods-The records of 178 women referred for colposcopy in 1993, with last smear showing BNC, were obtained from our laboratory database. The cytology, colposcopy, and biopsy follow up for a five year period were also obtained. The patients were divided into three categories according to their smear status before the last referral borderline smear: category 1 , persistent BNC (n = 39); category 2 , BNC preceded by dyskaryotic smears $(n=100)$; and category $3, \mathrm{BNC}$ after treatment for CIN $(n=39)$. The referral borderline smears were reviewed on cases with negative outcome and those with a biopsy diagnosis of CIN2 and CIN3.
\end{abstract}

Results-In 50 women (28\%) no biopsy was deemed necessary after colposcopic assessment. The biopsy results in the remaining $128(72 \%)$ women were as follows: normal in $18(\mathbf{1 0} \%)$, koilocytosis in $12(7 \%)$, CIN1 in $45(25 \%)$, CIN2 in 32 $(18 \%)$, and CIN3 in $21(12 \%)$ women. High grade lesions (CIN2, CIN3) were seen on biopsy in 14 of 39, 33 of 100 , and six of 39 cases in category 1 , category 2 , and category 3, respectively. Blind review of the referral borderline smears from 53 women with a biopsy diagnosis of high grade lesions (32 CIN2, 21 CIN3) confirmed they were borderline in 23 , upgraded them to mild dyskaryosis in 15, and found that $\mathbf{1 4}$ cases of isolated moderate or severe dyskaryotic cells had been missed originally. The borderline change was in mature squamous cells in five of 23 and in immature metaplastic epithelium in 18 of 23 cases. After smear review in 68 women with negative outcome, 36 smears were reclassified as negative in keeping with inflammation and atrophy, three were considered unsatisfactory, one was upgraded to CIN1, and 28 were confirmed as BNC. Of the latter, 25 of 28 were in mature squamous cells. The five year follow up on women with negative colposcopy $(n=50)$, negative loop excision of transformation zone (LETZ) $(n=18)$, and LETZ with koilocytosis $(n=12)$ showed subsequent high grade CIN on LETZ in 16,0 , and two patients, respectively.

Conclusions-On referral of women for colposcopy with last smear showing BNC, the outcome was high grade CIN in over $30 \%$ of cases, irrespective of whether the borderline smear was preceded by another borderline smear or by a dyskaryotic smear. In contrast, in those referred because of BNC after treatment of CIN, high grade CIN was seen less frequently ( $15 \%$ of cases). Furthermore, in cases that necessitated loop excisions, high grade CIN was seen in $41 \%$. This study also showed that BNC associated with inflammation or atrophy, or BNC in mature squamous cells, appears to have lower predictive value for CIN than those cases where BNC is associated with immature metaplastic epithelium. The use of terms such as "BNC favour reactive" for the former and "BNC favour dyskaryosis" for the latter is recommended, together with follow up by cytology and colposcopy, respectively.

(f Clin Pathol 2000;53:439-444)

Keywords: borderline nuclear abnormality; cervical cytology

The effectiveness of cervical screening programmes is measured by the reduction in the incidence of, and deaths from, cervical cancer. In an effort to categorise equivocal cases and minimise false negative results, laboratories utilise a term such as borderline nuclear changes (BNC). ${ }^{1-3}$ This is used for smears with nuclear abnormalities that cannot be ascribed with certainty to a benign process but are not sufficient for an unequivocal diagnosis of squamous dyskaryosis. In the Bethesda classification, the term "atypical squamous cells of uncertain significance (ASCUS)" is used for this purpose. ${ }^{4}$ However, the ASCUS classification excludes cases of human papillomavirus (HPV) infection, which form a large proportion of the cases classified as BNC. ${ }^{3}$ 
Table 1 Categories according to smear status before the last referral borderline smear

\begin{tabular}{lll}
\hline Category & Description & $n=178$ \\
\hline 1 & Those with at least 1 other borderline smear & $39(22.5 \%)$ \\
2 & Those with 1-2 mild or moderate dyskaryosis & $100(55 \%)$ \\
3 & Those who had treatment for CIN & $39(22.5 \%)$ \\
\hline
\end{tabular}

$\mathrm{CIN}$, cervical intraepithelial neoplasia.

Table 2 The outcome of women referred for colposcopy with $B N C$

\begin{tabular}{ll}
\hline Total No. & 178 women \\
\hline No biopsy taken & $50(28 \%)$ \\
Biopsy taken & $128(72 \%)$ \\
Biopsy negative & $18(10 \%)$ \\
Biopsy koilocytosis & $12(7 \%)$ \\
Biopsy CIN1 & $45(25 \%)$ \\
Biopsy CIN2 & $32(18 \%)$ \\
Biopsy CIN3 & $21(12 \%)$ \\
Biopsy CIN2,3 & $53(30 \%)$ \\
\hline
\end{tabular}

$\mathrm{BNC}$, borderline nuclear change; CIN, cervical intraepithelial neoplasia.

Nonetheless, the diagnosis of BNC/ASCUS has no clear cut morphological criteria. ${ }^{3}$ Inevitably, this category includes cases that ultimately prove to be inflammatory, reparative, or atrophic changes, as well as cases of cervical intraepithelial neoplasia (CIN) or the Bethesda equivalent SIL (squamous intraepithelial lesion). Borderline smear reports can form $5-20 \%$ of the workload of cervical cytology laboratories world wide, but the accepted standard in the UK is for BNC and mild dyskaryosis combined to be in the region of $5.5 \% .^{5}$ The optimal management of women with low grade abnormalities detected on smears remains controversial. The current practice in the UK is to request a repeat smear in 6-12 months for first and second BNC and a referral for colposcopy for the third one. For a first mild dyskaryosis, the recommendation is to repeat in three to six months and for a second to refer for colposcopy. ${ }^{2}$ Referral of women for colposcopy can cause high anxiety and unnecessary treatment with possible complications. ${ }^{6}$

There have been recent reports on the risk of CIN in women with smears showing BNC. Most of these reports suggest that CIN is found in a large proportion of subsequent smears or biopsies. ${ }^{7-15}$ Soofer and Sidawy found SIL on follow up smears in $24 \%$ of women with ASCUS compared with $3.9 \%$ in those with smears showing reactive changes and $1.6 \%$ of those with negative smears. ${ }^{14}$ Similarly, Stastny et al found a significant difference in the risk of developing SIL between women with a negative smear and those with an ASCUS smear $(6 \%$ v $24.3 \%) .^{15}$

The Edinburgh University department of pathology serves a population of approximately 250000 women in the cervical screening age

Table 3 Outcome of the three categories

\begin{tabular}{llllll}
\hline Category & $n$ & Negative & Koilcytosis & CIN1,2,3 & CIN2,3 \\
\hline & 178 & $68(44 \%)$ & 12 & $98(56 \%)$ & $53(28 \%)$ \\
1 Persistent BNC & 39 & $13(33 \%)$ & $5(13 \%)$ & $21(54 \%)$ & $14(36 \%)$ \\
2 BNC preceded by dyskaryosis & 100 & $32(32 \%)$ & $5(5 \%)$ & $63(63 \%)$ & $33(33 \%)$ \\
3 BNC after treatment for CIN & 39 & $23(59 \%)$ & $2(5 \%)$ & $14(44 \%)$ & $06(15 \%)$ \\
\hline
\end{tabular}

$\mathrm{BNC}$, borderline nuclear change; $\mathrm{CIN}$, cervical intraepithelial neoplasia. group (20-60 years) and receives over 85000 cervical smears and 2500-3000 cervical biopsies annually. The borderline smear category constitutes $5-7 \%$ of our workload, and nearly 200 to 300 women are referred annually for colposcopy after a BNC.

The aims of our study were: (1) to determine the outcome of women referred for colposcopy with smears showing BNC; (2) to find out whether the outcome is different depending on whether BNC was persistent, preceded by dyskaryosis, or followed treatment for CIN; and (3) to see whether a subtype of BNC can be identified that carries a higher predictive value for CIN.

\section{Materials and methods}

DATA COLLECTION

The records of 178 consecutive women referred for colposcopy at the Edinburgh Royal Infirmary in the year 1993, with a referral cervical smear showing BNC, were obtained from the laboratory database. Subsequent and prior biopsy and/or smear records with five year follow up were also obtained.

The patients were divided into three categories according to the smear history before the referral BNC (table 1): category 1, BNC preceded by at least one other borderline smear; category 2, BNC preceded by one or two dyskaryotic smears; and category $3, \mathrm{BNC}$ discovered at follow up after treatment for CIN.

SMEAR REVIEW

The referral borderline smears from 68 women who had negative outcome (either on colposcopy or biopsy) and from 53 women with a biopsy diagnosis of high grade CIN were selected for critical review independently (and blind to the colposcopic outcome) by two of the authors (GR and RAL). When the cellular changes were inconsistent with BNC, the smears were reclassified into negative with inflammatory or atrophic changes, or dyskaryotic. When BNC was confirmed, an attempt was made to classify it into either BNC in mature squamous cells or BNC in immature metaplastic cells.

\section{Results}

Table 2 shows the outcome of 178 women after referral for colposcopy. No colposcopic abnormality was found and biopsy was deemed unnecessary in 50 women $(28 \%)$. In the remaining 128 women, loop excision of transformation zone (LETZ) was performed. The biopsy diagnoses in this group of women were as follows: normal in $18(10 \%)$, koilocytosis in $12(7 \%)$, CIN1 in $45(25 \%)$, CIN2 in $32(18 \%)$, and CIN3 in $21(12 \%)$ women. CIN 2 and CIN 3 together constituted $30 \%$ of the total number of women (53 of 178) referred for colposcopy and $41 \%$ of those who had LETZ.

According to the smear history before the referral borderline smears, there were 39 women $(22.5 \%)$ in category 1 (those with at least one other borderline smear), 100 (55\%) in category 2 (those preceded by one or two 
Table 4 Review of 121 smears with BNC and comparison between those with negative outcome and those with biopsy results of CIN2,3

\begin{tabular}{llll}
\hline Review CC & 121 BNC & 68 negative outcome & 53 CIN2,3 smears \\
\hline BNC & $51(42 \%)$ & $28(41 \%)$ & $23(43 \%)$ \\
Negative & $36(30 \%)$ & $36(53 \%)$ & 0 \\
Mild dyskaryosis & $16(13 \%)$ & $1(2 \%)$ & $15(28 \%)$ \\
Mod/severe dyskaryosis & $14(12 \%)$ & 0 & $14(27 \%)$ \\
Unsatisfactory & $4(2 \%)$ & $3(4 \%)$ & $1(2 \%)$ \\
\hline
\end{tabular}

$\mathrm{BNC}$, borderline nuclear change; CIN, cervical intraepithelial neoplasia.

Table 5 Reasons for reclassifying BNC smears to negative

\begin{tabular}{ll}
\hline$n$ & 36 \\
\hline Inflammatory changed & $16(44 \%)$ \\
A few/bland nuclei only & $16(44 \%)$ \\
Atrophic smear & $4(11 \%)$ \\
\hline
\end{tabular}

BNC, borderline nuclear change.

dyskaryotic smears), and $39(22.5 \%)$ in category 3 (those in whom BNC followed treatment for CIN) (table 1). Table 3 shows the colposcopic and biopsy outcome of these three groups. A negative outcome was found in 13 of $39(33 \%)$ of those in category 1,32 of 100 $(32 \%)$ of those in category 2 , and in 23 of 39 $(59 \%)$ of those in category 3 . High grade CIN was found in 14 of $39(36 \%), 33$ of $100(33 \%)$, and six of $39(15 \%)$ of those in categories 1,2 , and 3 , respectively.

SMEAR REVIEW

Table 4 shows the results of reviewing 121 borderline smears from 68 women with negative colposcopic outcome and 53 women with biopsy diagnosis of high grade CIN. The left hand column shows the review results of these

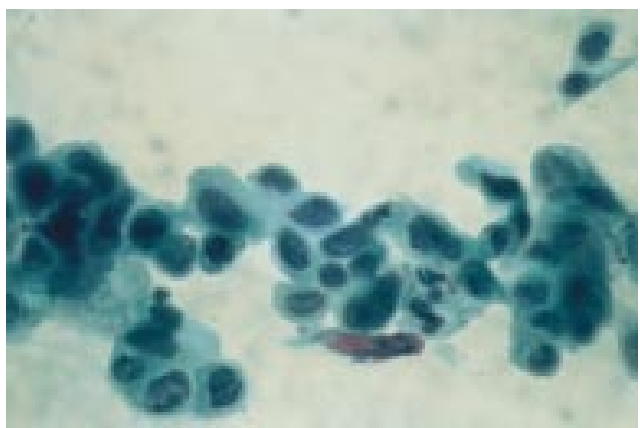

Figure 1 Isolated collection of moderately and severely dyskaryotic cells were misinterpreted as immature metaplastic cells with borderline nuclear change. Biopsy diagnosis cervical intraepithelial neoplasia 3.

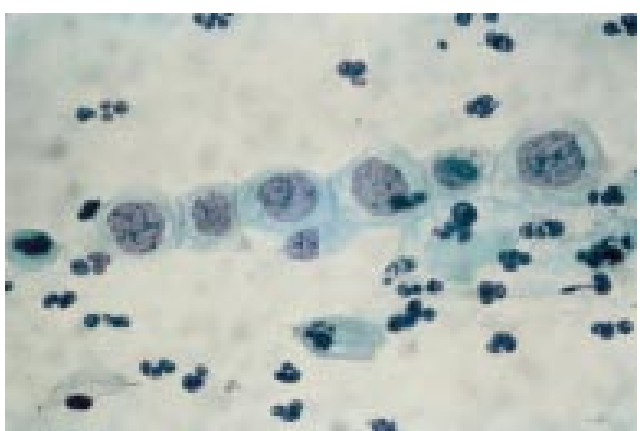

Figure 2 Isolated metaplastic cells showing enlarged nuclei with irregular borders, and course chromatin but no hyperchromasia (compared with adjacent polymorphs). These were reported as borderline nuclear change. Biopsy diagnosis cervical intraepithelial neoplasia 3.

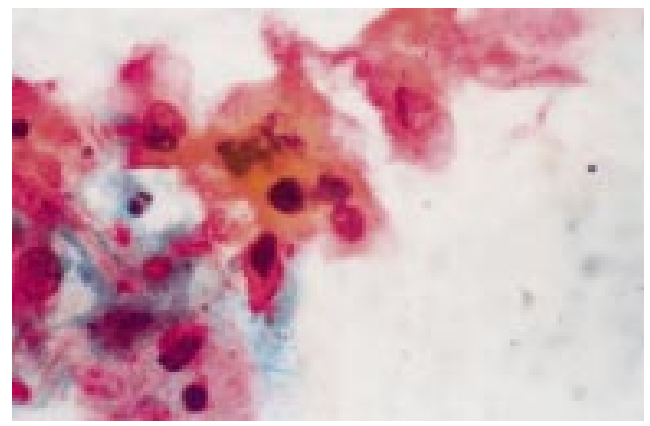

Figure 3 Nuclear enlargement, variation, and hyperchromasia in mature squamous cells, diagnosed as borderline nuclear change. Biopsy outcome with five years follow up is negative.

borderline smears. BNC was confirmed in 51 women $(42 \%)$, reclassified as negative in 36 (30\%), upgraded to mild dyskaryosis in 16 $(13 \%)$, and upgraded to moderate and severe dyskaryosis in $14(12 \%)$. Four smears (2\%) were reclassified as unsatisfactory. The middle column shows the smear review results of 68 women with negative colposcopic outcome or in whom biopsies were negative. In 28 women (41\%) BNC was confirmed, in 36 (53\%) the smears were reclassified as negative, in one $(2 \%)$ the smear was upgraded to mild dyskaryosis, and in three (4\%) smears were considered to be unsatisfactory. The right hand column shows the review results of 53 smears from women who had subsequent biopsy diagnosis of CIN2 and CIN3. The diagnosis of BNC was confirmed in $23(43 \%)$ smears, upgraded to mild dyskaryosis in 15 (28\%), considered unsatisfactory in one $(2 \%)$, and isolated moderately or severely dyskaryosis cells had been missed in $14(27 \%)$.

Table 5 shows the reasons for reclassifying 36 of 68 borderline smears as negative. In 16 of cases $(44 \%)$, the changes were considered to be inflammatory in nature, and in another 16 cases $(44 \%)$ they were thought to result from the presence of a few cells with enlarged nuclei or showing enlarged nuclei that lacked any chromatin abnormality. In the remaining four $(11 \%)$ the changes were the result of postmenopausal atrophy.

The smears that were upgraded to moderate or severe dyskaryosis were generally of poor quality and showed either a few dyskaryotic cells (fig 1), pale dyskaryosis (fig 2), or microbiopsy.

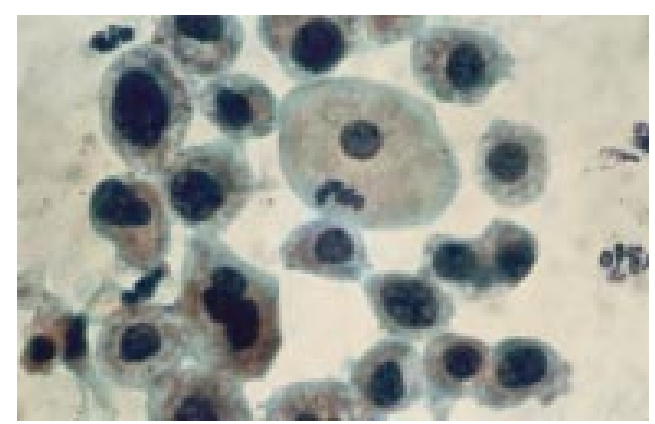

Figure 4 Nuclear enlargement and hyperchromasia in immature squamous cells diagnosed as borderline nuclear change. Biopsy outcome cervical intraepithelial neoplasia 3. 
Table 6 Five years follow up of 80 women with no biopsy, or a biopsy diagnosis of negative or koilocytosis

\begin{tabular}{lllll}
\hline & $n$ & $\begin{array}{l}\text { Persistent } \\
\text { BNC }\end{array}$ & $\begin{array}{l}\text { Persistent } \\
\text { dyskaryosis }\end{array}$ & $\begin{array}{l}\text { CIN2/3 } \\
\text { on biopsy }\end{array}$ \\
\hline No biopsy & $50(28 \%)$ & $3(6 \%)$ & $5(10 \%)$ & $4(8 \%)$ \\
Negative & $18(10 \%)$ & 0 & $1(6 \%)$ & 0 \\
Koilocytosis & $12(7 \%)$ & 1 & 0 & $2(17 \%)$ \\
\hline
\end{tabular}

BNC was confirmed in 51 smears, of which $30(59 \%)$ were in mature squamous cells (fig 3) and $21(41 \%)$ were in immature metaplastic cells (fig 4). When these subtypes were matched with the outcome, the following figures emerged: $\mathrm{BNC}$ in mature squamous cells was found in 25 of 28 (89\%) of those with negative outcome and in five of $23(22 \%)$ of those with high grade outcome. On the other hand, BNC in immature metaplastic cells was seen in three of $28(11 \%)$ and 18 of $21(78 \%)$ cases with negative and high grade CIN outcome, respectively.

SMEAR FOLLOW UPS

Table 6 illustrates the results of the five year cytology/biopsy follow up of 80 women who had negative outcome (no biopsy, 50; negative biopsy, 18; koilocytosis, 12). Persistent BNC was seen in three of $50(6 \%)$, dyskaryosis in five of $50(10 \%)$, and high grade CIN on further biopsy in four of $50(8 \%)$ cases with no biopsy on initial colposcopic assessment. Persistent dyskaryosis on smears was seen in only one of $18(6 \%)$ women who had negative biopsy results. Cervical intraepithelial neoplasia grade 2 was found on further biopsy in two of those $(17 \%)$ with an original biopsy diagnosis of koilocytosis.

\section{Discussion}

One of the greatest clinical dilemmas associated with cervical screening is the management of low grade cytological abnormalities, namely BNC and mild dyskaryosis. These abnormalities are of concern because in a small but important number, they reflect the presence of high grade CIN..$^{7-15}$ However, in contrast, many women are being treated unnecessarily, resulting in emotional stress and unjustifiably high costs to the National Health Service that could be used for other more pressing medical problems.

Our study has shown that none of our patients who had been referred because of repeated $\mathrm{BNC}$ had invasive disease, and nearly half had negative outcome. It also showed that irrespective of whether a borderline smear is preceded by another borderline smear, or by mildly or even moderately dyskaryotic cells, the outcome was high grade CIN in nearly one third of the cases. However, when borderline smears followed LETZ, high grade CIN was less common (15\% of cases). This study has also shown that not all women who were referred for colposcopy because of persistent BNC underwent loop excision. In 50 (28\%) of our patients no biopsy was taken because the cervix appeared normal at the time of initial referral for colposcopy. None of these women had invasive disease after five years of follow up, but $10 \%$ had subsequent dyskaryosis on further smears and $8 \%$ had biopsy diagnosis of high grade CIN (table 6). This might reflect the limitations of colposcopy in detecting CIN. Of the remaining 128 women who had a colposcopic abnormality that necessitated LETZ, 53 of 128 (41\%) did have high grade CIN on biopsy. This is almost identical to a recent study of 172 women who were referred for colposcopy because of persistent mild dyskaryosis or persistent borderline changes, $44 \%$ and $41 \%$ of whom were found to have high grade CIN, respectively (G Rebello, et al, 1999, personal communication). Other reports on the follow up of patients with ASCUS have yielded SIL rates ranging from $31 \%$ to $61 \% .{ }^{7-16}$ The high CIN rate after persistent BNC might reflect the fact the BNC is an extremely difficult diagnostic area and shows poor interobserver and intra-observer consistency. $^{317}$ In one report, ${ }^{18}$ five expert pathologists independently reviewed 200 smears previously classified as ASCUS. The use of Bethesda criteria resulted in reclassification of most cases as negative or SIL. However, not one smear was diagnosed as ASCUS by all five members, highlighting the difficulty in diagnostic reproducibility. However, despite the problems in consistency of diagnosis, we can conclude that persistent $\mathrm{BNC}$ is a predictor of high grade CIN in a considerable number of women. Nonetheless, whether we are underestimating some of the nuclear or cytological features of high grade dyskaryosis in favour of BNC remains to be seen. In our study, we upgraded borderline smears to CIN1 in $13 \%$ of cases, and moderate and severe dyskaryosis were missed in $10 \%$. It appears, therefore, that false negative smears are still a problem. Some of the reasons for missing high grade dyskaryosis in our study were: the presence of only a few abnormal cells, pale dyskarosis, and small microbiopsy like aggregates. These were often found in suboptimal smears. This problem has been highlighted recently by Mitchell and Medley ${ }^{19}$ and O'Sullivan et $a l,{ }^{20}$ who have emphasised the type of high grade smear that is likely to be reported incorrectly as negative under normal screening conditions. In a recent study, Bergeron and colleagues ${ }^{21}$ reviewed smear histories of 585 women with biopsy confirmed CIN3 and found $14.8 \%$ false negative smear diagnoses; on reviewing these smears, they found $41 \%$ to be abnormal, half of which were of high grade abnormality.

Does a subtype of BNC with more predictive value for high grade CIN exist? It has been shown that metaplastic squamous cells with $\mathrm{BNC} / \mathrm{ASCUS}$ carry a higher risk of CIN being detected on colposcopy and biopsy. ${ }^{22}{ }^{23}$ In our study, 18 of 23 (77\%) of our borderline smears with a biopsy outcome of high grade CIN showed atypical nuclei in immature squamous cells compared with three of $28(11 \%)$ of those with negative outcome. Bethesda workshop participants suggested initially that the diagnosis of ASCUS should be qualified as "favour reactive" or "favour squamous intraepithelial lesion (SIL)". The literature on this subject is 
varied but generally concludes that patients with ASCUS in whom a dysplastic process is favoured have a higher rate of SIL/cancer on follow up than do patients in whom a reactive process is favoured. ${ }^{23} \mathrm{~A}$ subcategorisation of ASCUS has been advocated with terms such as: "equivocal low grade SIL (LSIL)", "cannot rule out LSIL", "low grade potential", or "minimal changes" for cases where the most important abnormality is LSIL, and that might represent a reactive process or an early cytological manifestation of HPV infection. The terms "equivocal high grade SIL (HSIL)", "cannot rule out HSIL", "high grade potential", or "inconclusive" have been advocated for those cases that might be a high grade lesion or carcinoma, to distinguish them from the (more benign) equivocal LSIL category. ${ }^{24}$

In our study, we reclassified 36 of 68 (53\%) samples originally classified as $\mathrm{BNC}$ to negative (table 5). Of these, $16(44 \%)$ were associated with inflammation and four $(11 \%)$ with postmenopausal atrophy. In the remaining 16 (44\%), the nuclear abnormalities were seen in only a few cells and were in the form of enlarged nuclei lacking any chromatin abnormality. In the past, there was a category of smear report called "inflammatory changes" and the recommendation was early repeat smear. In their update, the British Society for Clinical Cytology and Royal College of Pathology representatives agreed to abolish this category in favour of either negative or borderline nuclear changes. ${ }^{3}$ We feel that many people are cautious and call what would previously have been called inflammatory change, BNC. This has resulted in a major increase in the size of the borderline category and a huge increase in referrals for colposcopy. The other problem is the atypical cells associated with postmenopausal atrophy (C Crum C, ES Cibas. 13th world congress of academic and environmental pathology, Nice, 1998). ${ }^{25-27}$ Smears from these women are usually air dried and show apparent nuclear enlargement, a pronounced inflammatory component, and clusters of small dark bare nuclei that are difficult to interpret. A helpful means of assessing atrophy related atypia is the "oestrogen test", whereby application of local oestrogen cream results in maturation of the squamous cells and easier interpretation of the cell morphology. However, the atypia may persist in spite of subsequent negative biopsy findings. ${ }^{28}{ }^{29}$ Abati and colleagues ${ }^{25}$ have found that nuclear hyperchromasia and irregularity in contours rather than nuclear enlargement alone seem to be the most reliable cytological criteria for the diagnosis of genuine dyskaryosis in atrophic smears. In countries where there are organised cervical screening programmes it is considered unethical to have a high proportion of false positive smears, which result in over-treatment and an unnecessary burden for women participating in cervical screening.

It is clear from these observations that some subtypes of borderline smears might have less predictive value for CIN than others. In view of this, we support the proposal of using phrases such as "BNC favour reactive", or "BNC favour low or high grade CIN". In this way, we could cut down considerably on the number of women referred unnecessarily for colposcopy by advising follow up by cytology only in the former category, and referral for colposcopy in the latter group. On applying these recommendations one would be in a position to assess prospectively the outcome of the various subtypes.

Further studies might be needed to provide more information to distinguish true precursor lesions from those that have no clinical relevance.

In summary, of all patients who were referred for colposcopy because of borderline smears, $38 \%(50+18=68)$ were referred unnecessarily, $7 \%$ (12 of 178) had koilocytosis, and the remaining $55 \%$ (98 of 178) had CIN, with $30 \%$ showing high grade lesions (CIN2 and CIN3). No women had invasive disease. Our study also emphasises the problem of false negative smears and identifies a group of apparent borderline changes that are truly dyskaryotic, and furthermore identifies a subgroup of BNC that is more predictive of high grade dyskaryosis.

1 Evans DMD, Hudson EA, Brown CL, et al. Terminology in gynaecological cytopathology: report of the working party of the BSCC. F Clin Pathol 1986;39:933-44.

2 Herbert A. Achievable standards, bench marks for reporting and criteria for evaluating cervical cytopathology: report of a working party set up by RCPath, BSCC and NHSCSP. Octo-
ber 1995, Publication No. 1 . Sheffield: NHSCSP Publications.

3 Buckley CH, Herbert A, Johnson J, et al. Borderline nuclear changes in cervical smears: guidelines on their recognition and management. F Clin Pathol 1994;47:481-92.

4 National Cancer Institute. National Cancer Institute workshop report: the 1988 Bethesda system for reporting cervical/vaginal cytology diagnoses. $7 A M A$ 1989;262: 931.

5 Pritchard J. Quality assurance guidelines for the cervical screening programme. Report of a working party convened by the NHS Cervical Screening Programme. NHSCP Publication

6 Jones MH, Singer A, Jenkins D. The mildly abnormal cervical smear - patients' anxiety and choice of management. $f$ $R$ Soc Med 1996;89:257-60.

7 Kohan S, Nouinoff J, Beckman EM, et al. Colposcopic screening of women with atypical Papanicolaou smears. $\mathcal{F}$ Reprod Med 1985;30:383-7.

8 Maier RC, Schultenover SJ. Evaluation of the atypical squamous cell Papanicolaou smear. Int $\mathcal{F}$ Gynecol Pathol 1986;5:242-8

9 Noumoff JS. Atypia in cervical cytology as a risk factor for intraepithelial neoplasia. Am f Gynecol 1987;156:628-31.

10 Davis GL, Hernandez E, Davis JL, et al. Atypical squamous cells in Papanicolaou smears. Obstet Gynecol 1987;69:436.

11 Jones DED, Creasman WT, Dombroski RA, et al. Evaluation of the atypical Pap smear. Am f Obstet Gynecol 1987; 157:544-9.

12 Morrison BW, Erickson ER, Doshi N, et al. The significance of atypical cervical smears. F Reprod Med 1988;33:809-12.

13 Kaminski PF, Stevens CW Jr, Wheelock JB. Squamous atypia on cytology: the influence of age. $f$ Reprod Med 1989;34:617-20.

14 Soofer SB, Sidawy MK. Reactive cellular change: is there an increased risk for squamous intraepithelial lesions? Cancer 1997;181:144-7.

15 Stastny JF, Remmers RE, London WB, et al. Atypical squamous cells of undetermined significance: a comparative review of original and automated rescreen diagnosis of cervical smears with long term follow-up. Cancer 1997;181: 348-53.

16 Selvaggi SM, Haefner HK. Reporting of atypical squamous cells of undetermined significance on cervical smears: is it significant? Diagn Cytopathol 1995;13:352-6.

17 Williams ML, Rimm DL, Pedigo MA, et al. Atypical squamous cells of undetermined significance: correlative histologic and follow-up studies from an academic medical centre. Diagn Cytopathol 1998;16:1-7.

18 Sherman ME, Schiffman MH, Lornice AT, et al. Toward objective quality assurance in cervical cytopathology. Am $\mathcal{f}$ Clin Pathol 1994;102:182-7.

19 Mitchell H, Medley G. Differences between Papanicolaou smears with correct and incorrect diagnosis. Cytopathology $1995 ; 6: 368-75$. 
20 O'Sullivan JP, A'Hern RP, Chapman PA, et al. A case-control study of true-positive versus false-negative
cervical smears in women with cervical intraepithelial neocervical smears in women with cervical intraepithe
plasia (CIN) III. Cytopathology 1998;9:155-61.

21 Bergeron C, Debaque H, Ayivi J, et al. Cervical smear histories of 585 women with biopsy-proven carcinoma in situ. Acta Cytol 1997;41:676-80.

22 Sheils LA, Wilbur DC. Atypical squamous cells of undetermined significance: stratification of the risk of association with, or progression to, squamous intraepithelial lesion based on morphologic subcategorization. Acta Cytol 1997; 41:1065-72.

23 Klin MI, Davey D. Atypical squamous cells of undetermined 1996 significance qualified: a follow-up study. Diagn Cytopathol 1996;14:380-4.

24 Solomon D, Frable W, Vooijs GP, et al. ASCUS and AGUS criteria: IAC task force summary. Acta Cytol 1998;42:1624 .
25 Abati A, Jaffurs W, Wilder AM. Squamous atypia in atrophic vaginal smear. Cancer (Cancer Cytology) 1998;84: $218-25$

26 Hirschowitz L, Raffle AK, Mackenzie EFD, et al. Long term follow up of women with borderline cervical smear-test results: effects of age and viral infection on progression to high grade dyskaryosis. BMF 1992;304:1209-12.

27 Medley G, Surtees VM. Squamous atypia in atrophic vaginal smear cancer [editorial counterpoint]. Cancer (Cancer Cytology) 1998;84:200-1.

28 Symmans F, Mechanic L, MacCormell P, et al. Correlation of cervical cytology and human papillomavirus DNA detection in postmenopausal women. Int f Gynecol Pathol 1992;11:204-9.

29 Kaminski PF, Sorosky JI, Wheelock JB, et al. The significance of atypical cervical cytology in an older population. Obstet Gynecol 1989;73:13-15. 